\title{
Criatividade e inteligência: analisando semelhanças e discrepâncias no desenvolvimento
}

\author{
Solange Muglia Wechsler \\ Pontificia Universidade Católica de Campinas \\ Maiana Farias Oliveira Nunes \\ Faculdade Avantis-SC \\ Patrícia Waltz Schelini \\ Universidade Federal de São Carlos \\ Adriana Aparecida Ferreira \\ Universidade Mogi das Cruzes \\ Dejenane Aparecida Pascoal Pereira \\ Prefeitura Municipal de São Sebastião-SP
}

\begin{abstract}
Resumo
As semelhanças e discrepâncias entre inteligência e criatividade foram investigadas, assim como os possíveis impactos de gênero e série educacional sobre o seu desenvolvimento. A amostra foi composta por 172 estudantes (91 mulheres, 81 homens), com idades variando dos 7 aos 17 anos. A avaliação da inteligência foi feita por meio da versão brasileira da Bateria Woodcock-Johnson III enquanto que criatividade foi avaliada pelos Testes de Pensamento Criativo de Torrance. Os resultados obtidos pelas MANOVA e ANOVA demonstraram efeitos significativos de série escolar para inteligência e criatividade figural, e de sexo para criatividade verbal. Não houve correlações significativas entre inteligência e criatividade, de acordo com as medianas nos testes de inteligência. A análise fatorial rotação Varimax apontou distinção entre inteligência, criatividade verbal e figurativa, indicando a independência entre estes construtos.
\end{abstract}

Palavras-chave: avaliação; testes; criatividade; inteligência; desenvolvimento cognitivo.

\begin{abstract}
Creativity and intelligence: analyzing developmental similarities and discrepancies. The similarities and discrepancies between intelligence and creativity were investigated as well as the impact of gender and educational level upon their development. The sample was composed of 172 students (91 women, 81 man), ages ranging from 7-17 years old. Intelligence was assessed through the Brazilian version of the WoodcockJohnson III tests, whereas creativity was evaluated through the Torrance tests of creativity. Results analyzed by MANOVA and ANOVA indicated significant effects of educational grade upon intelligence and creativity, and sex effects only on verbal creativity. No significant correlations were found between intelligence and creativity according to median results on intelligence tests. Factor analysis demonstrated the distinction among intelligence, verbal and figural creativity. On conclusion, creativity and intelligence were found as being independent constructs.
\end{abstract}

Keywords: assessment; tests; creativity; intelligence; cognitive development.

I nteligência e criatividade são fenômenos bastante estudados, embora até hoje exista bastante divergência quanto às suas definições (Furnham, Batey, Anand, \& Manfield, 2008; Kaufman, Plucker, \& Baer, 2008; Silvia, 2008). A inteligência tem sido compreendida sob diferentes aspectos, abrangendo não só as etapas envolvidas no desenvolvimento cognitivo, a natureza e dimensionalidade das habilidades que a compõem, como também os aspectos socioculturais que a influenciam (Gardner, 1999; McGrew, 2009; Sternberg, 1999; Sternberg \& Kaufman, 2001, Vincent, Decker, \& Munford, 2002). Por sua vez, a criatividade é discutida sob diferentes perspectivas, demonstrando ser um fenômeno de múltiplos aspectos, no qual interagem elementos cognitivos, características de personalidade, além das variáveis de natureza familiar, educacional e social (Torrance \& Safter, 1999; Torre \& Violant, 2006; Wechsler, 2009).

As controvérsias sobre as possíveis relações entre criatividade e inteligência existem há mais de 40 anos (Getzels \& Jackson, 1963; Wallach \& Kogan,1965). Os questionamentos referem-se à dimensionalidade destes construtos (Guilford \& 
Christensen, 1973; Puccio \& Murdock, 1999), analisando se estes seriam sinônimos ou conceitos sobrepostos, existindo um limiar no qual poderia ser esperada uma alta relação entre ambos. McKinnon (1978), por exemplo, ao comparar arquitetos e pesquisadores criativos, observou que, embora $98 \%$ tivessem um Coeficiente de Inteligência (QI) superior a $120 \mathrm{em}$ testes de inteligência, eles não necessariamente alcançavam pontuação alta em testes de criatividade. Por sua vez, no estudo de Runco e Albert (1986), realizado com crianças, foi demonstrado que somente existiam relações significativas entre alta inteligência e criatividade, enquanto que em outros níveis intelectuais não foram encontradas relações significativas - fato também confirmado no estudo de Preckel, Holling, e Wiese (2006), com estudantes de Ensino Médio. Entretanto, debates sobre os limiares de relações entre esses conceitos são pouco conclusivos, como afirma Runco (2007), ao revisar estudos na área.

Segundo a formulação de Cattell (1971), a inteligência poderia ser entendida de acordo com duas grandes dimensões: Fluida (capacidade de raciocínio) e cristalizada (conhecimento adquirido no ambiente). Uma compreensão mais ampla do funcionamento intelectual foi desenvolvida por McGrew (2005, 2009), ao combinar as propostas teóricas de Cattell-HornCarroll, em um modelo conhecido como CHC. De acordo com a proposição do $\mathrm{CHC}$, a inteligência poderia ser entendida por meio de três camadas, estando na primeira cerca de 70 habilidades específicas, na segunda 10 habilidades amplas e na terceira um possível fator Geral (McGrew \& Flanagan, 1998). Dentre os poucos instrumentos disponíveis para avaliar a inteligência, segundo esse modelo, encontra-se a Bateria Woodcock-Johnson III (Woodcock, McGrew, \& Mather, 2001). Embora não exista uma definição para criatividade dentro desse modelo, considerase que a habilidade de recuperação em longo prazo represente a associação entre ideias (avaliada pelos subtestes de memória auditiva visual e memória visual adiada), podendo a criatividade ser mensurada por meio dessa bateria (McGrew \& Woodcock, 2001; Schrank, 2005).

Estudos da adaptação e validação da Bateria WoodcockJohnson têm sido realizados com crianças e jovens brasileiros. Na pesquisa realizada por Wechsler e Schelini (2006), com estudantes dos 7 aos 17 anos, foram observados ganhos cognitivos nas habilidades medidas pelos subtestes dessa bateria. Por sua vez, diferenças de sexo somente foram observadas em algumas faixas etárias, nas habilidades de pensamento visoespacial e raciocínio fluido, indicando resultados superiores do sexo feminino nas primeiras séries (7-9 anos) sendo posteriormente igualadas ou superadas pelo sexo masculino. A confirmação de acréscimos no desenvolvimento cognitivo de crianças e jovens, da mesma faixa etária, foi obtida em um estudo posterior por Wechsler et al. (no prelo), com uma amostra de 1.097 estudantes, no qual foi demonstrada a validade de uma versão brasileira, adaptada para essa bateria.

Dentre as medidas de criatividade mais utilizadas, em nível internacional, destacam-se os Testes de Pensamento Criativo de Torrance (Torrance, 1966, 1990) em suas formas verbal e figurativa (Raina, 2006). Enquanto que nas primeiras publicações desses testes existia um foco em quatro características do pensamento divergente (Fluência, Flexibilidade, Originalidade e
Elaboração), nas suas mais recentes edições foram incorporadas outras 13 características criativas envolvendo não só aspectos cognitivos, mas também emocionais da criatividade (Torrance \& Ball, 1984; Torrance, Ball, \& Safter, 1990). Devido à sua composição, esses testes são considerados as medidas mais completas para se avaliar a criatividade. Traduções e validações do mesmo são encontradas em mais de 33 países (Kaufman \& Baer, 2006). Uma versão brasileira foi desenvolvida e validada por Wechsler (2006).

O desenvolvimento da criatividade de crianças e jovens tem sido bastante estudado por meio de testes, como aponta a revisão do estado da área nas publicações brasileiras (Nakano \& Wechsler, 2007). Nos trabalhos de normatização dos testes de criatividade figural e verbal de Torrance, foram observadas diferenças de sexo e idade nesses instrumentos (Wechsler, 2004a, 2004b). Nesses dois tipos de testes, o sexo masculino obteve resultados melhores no nível de Ensino Médio, enquanto que essa tendência se reverteu no Ensino Superior (Nakano \& Wechsler, 2006). Entretanto, ainda são escassos os estudos brasileiros comparando a inteligência com a criatividade. Por exemplo, no estudo de Wechsler e Richmond (1984), realizado com crianças, foram demonstradas fracas relações, não significativas, entre inteligência e criatividade. Por sua vez, o trabalho de Aranha (1997) indicou que existiam relações entre criatividade e inteligência somente no nível socioeconômico médio-alto, não ocorrendo, entretanto, na classe econômica mais desfavorecida.

Considerando a necessidade de maior conhecimento sobre inteligência e criatividade, este estudo visou investigar as semelhanças e diferenças entre esses construtos. Outro foco de análise foram as influências de gênero e série educacional sobre o desenvolvimento intelectual e criativo de crianças e jovens.

\section{Método}

\section{Participantes}

A amostra foi composta por 172 estudantes (91 mulheres e 81 homens), estudantes de escolas privadas $(49,4 \%$ ) e públicas $(50,6 \%)$, localizadas em cinco cidades no interior do estado de São Paulo. Esses estudantes cursavam da $1^{\underline{\underline{a}}}$ série do Ensino Fundamental até o $3^{\underline{0}}$ ano do Ensino Médio. As faixas etárias envolvidas foram classificadas da seguinte maneira: 7-9 anos (36); 10-12 anos (36); 13-15 anos (56); 16-18 anos (43). As escolas de cada cidade foram selecionadas de acordo com o critério de conveniência do pesquisador local, existindo em torno de 32 crianças por cidade.

\section{Instrumentos}

Woodcock-Johnson III Tests of Cognitive Abilities-WJIII (Woodcock et al., 2001). Esta bateria é composta por 10 subtestes que avaliam diferentes habilidades intelectuais. Os testes utilizados foram: 1) Compreensão e conhecimento (Gc- linguagem e inteligência cristalizada), composto pelos subtestes de Vocabulário, Sinônimos, Antônimos e Analogias; 2) Aprendizagem visual auditiva (Glr- memória de associações); 3) Relações espaciais (Gv- pensamento viso-espacial); 4) Combinação de sons (Ga- análise e síntese de sons); 5) Formação 
de conceitos (Gf- raciocínio lógico ou inteligência fluida); 6) Combinações visuais (Gs- rapidez de processamento); 7) Números invertidos (Gsm- memória de trabalho ou curto prazo); 8) Palavras incompletas (Ga- memória auditiva); 9) Memória de trabalho auditiva (Glr- memória e atenção dividida); e 10) Aprendizagem visual auditiva adiada (Glr- memória associativa ou de recuperação). O desempenho nestes subtestes pode ser analisado de forma isolada, pois representam diferentes habilidades envolvidas na inteligência, ou por meio de um escore total, na medida em que representa um fator geral, no modelo CHC.

A validade da versão brasileira da WJ-III foi comprovada em vários estudos. A adequação dos itens brasileiros criados para esta bateria foi demonstrada pela Teoria de Resposta ao Item, indicando assim a necessidade de uma versão adaptada para a parte verbal desta bateria (Wechsler, Vendramini, \& Schelini; 2007; Wechsler et al., no prelo). A validade de critério da bateria foi observada na pesquisa de Mol e Wechsler (2008), ao ser constatado que grupos de crianças com e sem dificuldades de aprendizagem poderiam ser identificados pelo seu desempenho na Bateria. Por sua vez, a validade convergente da versão brasileira foi comprovada ao serem observadas altas correlações entre os seus resultados e aqueles obtidos na Escala de Inteligência Wechsler para Crianças - WISC-III (Chiodi \& Wechsler, 2009).

Testes de pensamento criativo de Torrance, versões figural e verbal, forma A (Torrance, 1966, 1990). O teste de pensamento criativo de Torrance, na sua versão figural, é composto por três atividades contendo rabiscos a serem completados. $\mathrm{Na}$ versão verbal existem seis atividades, para as quais são solicitadas perguntas, causas, consequências ou ideias para melhoria de produtos. Estes dois testes são corrigidos de acordo com características ou indicadores cognitivos e emocionais relacionados com a criatividade, identificados nas pesquisas relacionadas com a pessoa criativa, em estudos internacionais e nacionais (Torrance \& Safter, 1999; Wechsler, 2004a, 2004b).

Os indicadores cognitivos, relacionados ao pensamento divergente, são os seguintes: Fluência (capacidade de produzir grande número de ideias), Flexibilidade (propostas de diferentes formas ou pontos de vistas), Elaboração (detalhamento ou enriquecimento da ideias para torná-las mais vívidas), e Originalidade (soluções incomuns ou fora dos padrões). Os indicadores emocionais que podem ser identificados na criatividade são: Emoção (expressão de sentimentos), Fantasia (representação de mundos imaginários), Movimento (dinâmica nas ações), Perspectiva Incomum (visão sob diferentes perspectivas), Perspectiva Interna (representação de partes de algo oculto); Uso de Contextos (preocupações com o ambiente); Combinação (síntese de ideias), Extensão de Limites (quebra de restrições); Títulos Expressivos (ir além da descrição). Estes 13 indicadores, cognitivos e emocionais, podem ser encontrados na criatividade figurativa (Torrance et al., 1990). Na criatividade verbal, oito indicadores podem ser encontrados: Fluência, Flexibilidade, Elaboração, Originalidade, Emoção, Fantasia, Perspectiva Incomum e Analogias/Metáforas (Wechsler, 2006). Uma medida mais completa para avaliação da criatividade pode ser obtida a partir da composição das características, tendo-se assim um Índice criativo I (composto por Fluência, Flexibilidade, Originalidade e Elaboração) e um Índice Criativo II (adicionando todas as características cognitivas e emocionais).

\section{Procedimento}

Diretores de escolas de Ensino Fundamental e Médio foram contatados por pesquisadores de diferentes cidades, de acordo com o critério de conveniência. Após autorização destes, foram enviados os termos de permissão para pais das crianças solicitando a participação de seu filho na pesquisa, que seria aplicada em horário extraclasse. As cartas de permissão recebidas foram sorteadas, de forma aleatória, de acordo com a faixa etária da criança.

As crianças foram avaliadas em duas sessões, individualmente. Na primeira sessão foram aplicados todos os testes do WJ-III, com exceção do teste 10 , que requer o intervalo de uma semana após a $1^{\underline{a}}$ aplicação. Além destes, o teste de criatividade figural de Torrance foi aplicado no $1^{\underline{0}}$ momento. Na segunda sessão foram administrados os testes de criatividade verbal de Torrance e o subteste $10 \mathrm{da}$ WJ-III.

Os resultados foram trabalhados a partir dos escores brutos em inteligência e criatividade. Primeiramente, foram analisadas as influências de sexo e série educacional nos resultados totais da WJ, e nos índices criativos verbal e figural. A idade foi mantida como covariável devido às diferenças existentes de faixas etárias nas séries escolares. O nível socioeconômico não entrou no modelo, considerando que não havia representatividade para todas as séries estudadas. As análises Multivariada e Univariada da Variância (MANOVA, ANOVA) foram utilizadas para este estudo. Posteriormente, as correlações de Pearson foram empregadas com os grupos divididos de acordo com a pontuação mediana no resultado final da WJ-III, a fim de verificar se existiriam associações entre inteligência e criatividade, em diferentes limiares. A análise fatorial Varimax foi realizada para verificar a independência entre os construtos de criatividade e inteligência, tendo como critérios a carga fatorial acima 0,30 , eigenvalue $\geq 2,0$ e o gráfico de dispersão dos fatores.

\section{Resultados}

Os resultados no desempenho total dos subtestes de inteligência, índices criativos figurais e verbais demonstraram ser altamente influenciados por série educacional quando analisados pela MANOVA $(p \leq 0,001)$. Devido a este fato, estes resultados foram analisados mais detalhadamente pela ANOVA, como demonstrado na Tabela 1.

As influências de faixa etária (covariável), sexo, série e interações sobre as medidas de inteligência e criatividade estão apresentadas na Tabela 1. Como pode ser verificado, o efeito significativo de série foi observado para a Bateria de Inteligência WJ. Ao comparar as médias por série na pontuação total desta bateria, pode-se compreender estes resultados. No Ensino Fundamental, as médias obtidas foram as seguintes: $1^{\underline{a}}$ série $=$ 358,$5 ; 2^{\underline{a}}$ série $=393,1 ; 3^{\underline{a}}$ série $=441,1 ; 4^{\underline{a}}$ série $=411,8 ; 5^{\circ}$ série $=396,7 ; 6^{\underline{a}}$ série $=428,7 ; 7^{\underline{a}}$ série $=481,4 ; 8^{\underline{a}}$ série $=467,9$. No Ensino Médio, as médias por ano foram: $1^{\circ}$ ano $=468,7 ; 2^{\circ}$ ano 
Tabela 1

Análise da Variância nos resultados totais em inteligência, criatividade verbal e figural

\begin{tabular}{lcccccccc}
\hline & \multicolumn{2}{c}{ Faixa etária $^{\mathrm{a}}$} & \multicolumn{2}{c}{ Sexo $^{\mathrm{b}}$} & \multicolumn{2}{c}{ Série $^{\mathrm{c}}$} & \multicolumn{2}{c}{ Sexo x Série $^{\mathrm{d}}$} \\
\cline { 2 - 9 } Subtestes & $F$ & Eta $^{2}$ & $F$ & Eta $^{2}$ & $F$ & Eta $^{2}$ & $F$ & Eta $^{2}$ \\
\hline Total WJ & 1,686 & 0,014 & 1,496 & 0,010 & $2,493^{*}$ & 0,146 & 0,904 & 0,055 \\
Indic. Verb I & 0,446 & 0,003 & $4,260^{*}$ & 0,028 & 1,003 & 0,087 & 1,235 & 0,108 \\
Indic. Verb. II & 0,422 & 0,003 & 1,810 & 0,011 & 0,749 & 0,073 & 1,042 & 0,092 \\
Indic. Figural I & 0,119 & 0,000 & 1,526 & 0,006 & $2,350^{*}$ & 0,138 & 0,646 & 0,035 \\
Indic. Figural II & 0,940 & 0,005 & 1,463 & 0,006 & $2,419 *$ & 0,140 & 0,756 & 0,035 \\
\hline
\end{tabular}

Nota. ${ }^{\mathrm{a}, \mathrm{b}} 1$ grau de liberdade; ${ }^{\mathrm{c}, \mathrm{d}} 10$ graus de liberdade.

${ }^{*} p \leq 0,05 ; * * p \leq 0,01 ; * * * p \leq 0,001$.

$=507,9 ; 3^{0}$ ano $=492,8$. Assim, os ganhos foram contínuos com o avanço das séries apesar de pequenas quedas.

Nos Índices Criativos Figurais I e II também foram observados ganhos significativos por séries. No Ensino Fundamental, as médias obtidas no Índice Figural I foram: $1^{\mathrm{a}}$ série $=21,27 ; 2^{\underline{a}}$ série $=22,1 ; 3^{\underline{a}}$ série $=19,7 ; 4^{\underline{a}}$ série $=29,1 ; 5^{\underline{a}}$ série $=36,5 ; 6^{\underline{a}}$ série $=31,5 ; 7^{\underline{a}}$ série $=43,2 ; 8^{\underline{a}}$ série $=37,6$. No Ensino Médio, as médias por ano foram: $1^{\underline{0}}$ ano $=46,2 ; 2^{\underline{0}}$ ano $=39,9 ; 3^{0}$ ano $=42,5$. Em relação ao Índice Criativo Figural II, as médias obtidas por série foram as seguintes: $1^{\underline{a}}$ série $=$ 20,$1 ; 2^{\underline{a}}$ série $=29,4 ; 3^{\underline{a}}$ série $=27,1 ; 4^{\underline{a}}$ série $=32,5 ; 5^{\underline{a}}$ série $=$ 42,$6 ; 6^{\mathrm{a}}$ série $=48,2 ; 7^{\mathrm{a}}$ série $=51,1 ; 8^{\mathrm{a}}$ série $=40,7$. No Ensino Médio, as médias por ano foram: $1^{\underline{0}}$ ano $=38,2 ; 2^{\underline{0}}$ ano $=39,9$; $3^{\underline{0}}$ ano $=42,9$. Podemos observar que, apesar de pequenos decréscimos, existiram ganhos nestes índices na medida em que a série aumentava.

Quanto à influência de sexo do participante, esta somente foi observada para o Índice Criativo Verbal I. O sexo feminino obteve pontuação média $(80,1)$ significativamente maior do que o masculino $(66,86)$ nesta variável.

Considerando as influências de sexo e série sobre as medidas de inteligência e criatividade, foram utilizados os resíduos derivados das ANOVAS para realizar as correlações entre as mesmas. Os resultados foram analisados com a amostra total e pela divisão de grupos, acima ou abaixo da mediana do escore total na WJ-III, como demonstrado na Tabela 2.

Tabela 2

Correlação residual entre o total da WJ-III e os Índices criativos figurais e verbais

\begin{tabular}{|c|c|c|c|c|c|}
\hline Índices Criativos & Total WJ-III & $\begin{array}{l}\text { Índ. Criativo } \\
\text { Figural I }\end{array}$ & $\begin{array}{c}\text { Índ. Criativo } \\
\text { Figural II }\end{array}$ & $\begin{array}{c}\text { Índ. Criativo } \\
\text { Verbal I }\end{array}$ & $\begin{array}{c}\text { Índ. Criativo } \\
\text { Verbal II }\end{array}$ \\
\hline \multicolumn{6}{|l|}{ Amostra Total WJ-III } \\
\hline Total WJ-III & - & $0,223 * *$ & $0,204 * *$ & 0,127 & 0,061 \\
\hline Índ. Criativo Figural I & & - & $0,952 * *$ & $0,291 * *$ & $0,303 * *$ \\
\hline Índ. Criativo Figural II & & & - & $0,332 * *$ & $0,357 * *$ \\
\hline Índ. Criativo Verbal I & & & & - & $0,882 * *$ \\
\hline Índ. Criativo Verbal II & & & & & - \\
\hline \multicolumn{6}{|l|}{ Abaixo mediana WJ } \\
\hline Total WJ-III & - & 0,115 & 0,056 & 0,094 & 0,049 \\
\hline Índ. Criativo Figural I & & - & $0,955^{* *}$ & $0,331 * *$ & $0,336 * *$ \\
\hline Índ. Criativo Figural II & & & - & $0,319 * *$ & $0,327 * *$ \\
\hline Índ. Criativo Verbal I & & & & - & $0,984 * *$ \\
\hline Índ. Criativo Verbal II & & & & & - \\
\hline \multicolumn{6}{|l|}{ Acima mediana WJ } \\
\hline Total WJ-III & - & 0,122 & 0,073 & 0,134 & 0,095 \\
\hline Índ. Criativo Figural I & & - & $0,954 * *$ & $0,332 * *$ & $0,337 * *$ \\
\hline Índ. Criativo Figural II & & & - & $0,326 * *$ & $0,334 * *$ \\
\hline Índ. Criativo Verbal I & & & & - & $0,984 * *$ \\
\hline Índ. Criativo Verbal II & & & & & - \\
\hline
\end{tabular}

De acordo com a Tabela 2, existem correlações significativas ( $p \leq 0,01)$ entre inteligência e criatividade figural quando se considera a amostra geral, o que não ocorre com a criatividade verbal. Entretanto, ao se considerar as correlações entre os grupos com desempenho acima ou abaixo da mediana na Bateria WJIII, as correlações entre inteligência e criatividade são muito baixas, não alcançando significância estatística. Deve ser também observado que as correlações entre as medidas de criatividade figural e verbal são altamente significativas, tanto para a amostra geral quanto para os grupos com resultados abaixo ou acima da mediana em inteligência.

O aprofundamento da análise entre inteligência e criatividade foi realizado por meio da Análise Fatorial, rotação Varimax, com os totais das pontuações totais nos diferentes subtestes da Bateria WJ-III e nos indicadores de criatividade figural e verbal. O teste de hemisfericidade de Bartlett indicou um valor significativo de $p$ $\leq 0,0001\left(X^{2}=2736,83, \mathrm{gl}=561\right)$ demonstrando a adequação da análise. Os resultados encontrados indicaram uma composição de 
quatro fatores, com variância total explicada de $46,04 \%$ (fator 1 $=17,21 \%$; fator $2=12,29 \%$; fator $3=9,28 \%$; e fator $4=7,27 \%$ ). A composição final dos fatores está representada na Tabela 3.

A análise dos componentes fatoriais, apresentada na Tabela 3 , indica que o primeiro fator é exclusivamente composto pelos subtestes de inteligência, agrupados entre si. O segundo fator é composto, basicamente, pela criatividade verbal, ao passo que os fatores 3 e 4 agrupam características relacionadas com a criatividade figurativa.

No primeiro fator são encontrados os testes de inteligência cristalizada (analogias, sinônimos, antônimos, vocabulário), de memória (visual auditiva e adiada), de processamento auditivo (palavras incompletas, combinação de sons) e de inteligência fluída (formação de conceitos). No segundo fator são encontradas todas as características criativas verbais, uma característica criatividade figural (títulos expressivos nos desenhos) além de um único subteste de inteligência (combinação visual, que mede a rapidez de processamento). O terceiro fator é composto, basicamente, dos componentes cognitivos da criatividade figural (fluência, flexibidade, originalidade) acrescido de um indicador criativo figural (extensão de limites). O quarto fator é composto de seis indicadores de criatividade figural (elaboração, movimento, perspectiva interna, emoção, fantasia, contexto), além de um único subteste de inteligência viso-espacial.

Tabela 3

Análise fatorial entre inteligência, criatividade figural e verbal

\begin{tabular}{|c|c|c|c|c|}
\hline \multirow[b]{2}{*}{ Subtestes } & \multicolumn{4}{|c|}{ Componentes } \\
\hline & 1 & 2 & 3 & 4 \\
\hline Analogias & 0,831 & & & \\
\hline Sinônimos & 0,780 & & & \\
\hline Antonimos & 0,756 & & & \\
\hline Ap.Vis. Aud. Adiada & 0,686 & & & \\
\hline Vocabulário & 0,674 & & & \\
\hline AprendizVisual Aud. & 0,618 & & & \\
\hline Formação Conceitos & 0,597 & & & \\
\hline Combinação. Sons & 0,567 & & & \\
\hline Memória Trab. Aud. & 0,561 & & & \\
\hline Palavr. Incompletas & 0,529 & & & \\
\hline Flexibilid. Verbal & & 0,853 & & \\
\hline Fluência Verbal & & 0,783 & & \\
\hline Originalidad. Verbal. & & 0,724 & & \\
\hline Persp.Incom.Verbal & & 0,621 & & \\
\hline Fantasia Verbal & & 0,449 & & \\
\hline Comb. Visual WJ & & 0,427 & & \\
\hline Emoção Verbal & & 0,420 & & \\
\hline Títulos Expr. Figural & & 0,365 & & \\
\hline Elabora. Verbal & & 0,337 & & \\
\hline Analogia Verbal & & 0,334 & & \\
\hline Fluência Figural & & & 0,881 & \\
\hline Flexibil. Figural & & & 0,849 & \\
\hline Original. Figural & & & 0,798 & \\
\hline Ext. Limites Figural & & & 0,687 & \\
\hline Elabor. Figural & & & & 0,738 \\
\hline Movimento figural & & & & 0,665 \\
\hline Persp. Interna Figur & & & & 0,605 \\
\hline Contexto Figural & & & & 0,467 \\
\hline Viso-espacial WJ & & & & 0,466 \\
\hline Emoção figural & & & & 0,456 \\
\hline Fantasia figural & & & & 0,441 \\
\hline
\end{tabular}

\section{Discussão e conclusões}

As relações entre inteligência e criatividade são focos de debates entre os estudiosos da área, questionando se estes construtos são semelhantes ou se podem ser explicados de forma distinta (Runco, 2007). Neste estudo, esses temas foram investigados por meio de testes psicológicos já validados no país a fim de obter informações mais amplas sobre esses conceitos. Com este propósito, foram escolhidos, como medida de inteligência, a Bateria Woodcock-Johnson III (Woodcock et al., 2001), e de criatividade, os testes de criatividade verbal e figural de Torrance (Torrance, 1990). As influências de sexo e série educacional sobre o desenvolvimento da inteligência e criatividade foram alvos deste estudo.

A série educacional, que reflete também as mudanças na faixa etária, demonstrou exercer considerável impacto no desenvolvimento das habilidades intelectuais. Confirmando estudos anteriores feitos no país com a Bateria WJ (Wechsler et al., no prelo), houve acréscimo nas habilidades cognitivas ao serem comparados estudantes do Ensino Fundamental e 
Médio. Entretanto, não foram observadas diferenças entre os sexos na pontuação total em inteligência. Tais resultados estão em consonância com estudos internacionais ao indicar que, embora existam pequenas diferenças em algumas das habilidades intelectuais entre homens e mulheres, os resultados totais em inteligência não demonstram diferenças significativas de gênero (Camarata \& Woodcock, 2006; Keith, Reynolds, Patel, $\&$ Ridley, 2008). Tais resultados demonstram a validade desta Bateria para o país.

O impacto da série educacional foi observado nas duas medidas de criatividade, verbal e figurativa. Ficou demonstrado que existe um aumento do potencial criativo até a $5^{\mathrm{a}}-6^{\mathrm{a}}$ série do Ensino Fundamental, sendo seguido por um decréscimo após esse período. Essa queda existe para os dois sexos, principalmente em criatividade verbal, para o sexo masculino. Os efeitos da escola sobre a criatividade têm sido alvo de vários estudos no país, indicando que o seu papel, infelizmente, tem sido mais o de limitar do que estimular a criatividade (Alencar, 2007; Alencar \& Fleith, 2008; Beauchamp, Merle Karnes, \& Johnson, 1993). Estes resultados vêm confirmar a necessidade de investimento na capacitação de professores para que possam desenvolver estratégias que facilitem pensar criativamente sobre as informações dadas, ao contrário do excesso de memorização que têm predominado nas escolas (Fleith \& Alencar, 2005, 2006; Martinez, 2002). Por sua vez, mulheres têm recebido maior atenção nas escolas, por possuírem um estilo de aprendizagem mais relacionado com o emocional, o que pode afetar os resultados dos estudantes do sexo masculino, e desencorajá-los na expressão criativa (Siqueira \& Wechsler, 2009).

As relações entre inteligência e criatividade encontradas neste estudo foram significativas somente para a amostra geral. Porém, este fato não se confirmou quando os grupos foram subdivididos segundo os resultados acima ou abaixo da mediana na WJ, existindo correlações baixas e não significativas entre inteligência e criatividade. Tal fato confirma os resultados de outros estudos (por exemplo, Davis \& Belcher, 1999; Kim, 2006, Runco, 2007) que relataram a existência de correlações baixas ou até negativas entre esses construtos. Percebe-se, portanto, que a criatividade pode ser encontrada em qualquer nível de inteligência, devido a sua fraca relação com a mesma. A relação significativa entre os índices criativos verbais e figurais encontrados confirmam a validade convergente desses construtos, indicando que a criatividade pode estar presente em diversas áreas, tal como já apontado por Wechsler (2004a, 2004b).

A independência dos construtos de inteligência e criatividade foi verificada neste estudo. Assim, demonstrou-se que a inteligência pertence a um fator ou construto separado da criatividade verbal e figurativa. $\mathrm{Na}$ dimensão intelectual, os fatores de inteligência cristalizada ou compreensão verbal (diretamente influenciados pela escola e ambiente sociocultural) estão combinados com outros, que envolvem o processamento auditivo, a memória e a inteligência fluida (ou o pensamento lógico), confirmando a importância desses aspectos, segundo diversos autores (Cattell, 1971, McGrew, 2009). Por sua vez, a criatividade verbal demonstra ter grande impacto na maneira como esses componentes se agrupam, abrangendo as características cognitivas, bem como as emocionais, sendo também influenciada pela rapidez de processamento da resposta. A criatividade figurativa aparece, a seguir, como sendo composta por dois fatores, representados por indicadores cognitivos e emocionais. A habilidade viso-espacial também está presente no último fator indicando a sua importância na avaliação da criatividade por desenhos.

A recuperação da informação (avaliada nos testes de memória visual auditiva e adiada na WJ-III), considerada parte da criatividade por representar a associação entre ideias (Schrank, 2005), não se agregou aos fatores de criatividade, mas sim no primeiro fator da inteligência. Este resultado seria esperado para os autores que compreendem a criatividade de maneira mais ampla, do que a mera associação de informações pela memória, pois nela estariam envolvidos não só componentes cognitivos, mas também afetivos, educacionais e socioculturais (Giglio, Wechsler, \& Bragotto, 2009; Morais \& Bahia, 2008; Torrance \& Safter, 1999; Torre \& Violant, 2006).

A inteligência e a criatividade são aspectos essenciais do funcionamento cognitivo. Esta pesquisa demonstrou que estes construtos são independentes e que não existe um limiar intelectual no qual a criatividade poderia ser esperada com maior ou menor intensidade (Munford, 2001). Confirmam-se, mais uma vez, as recomendações de Torrance (1965), ao indicar que todos nós possuímos potencial criativo, bastando apenas desenvolvêlo. Entretanto, ainda são necessárias mais pesquisas para investigar a relação entre criatividade e inteligência em processos de inovação, considerando a relevância destes construtos quando aplicados aos diferentes campos de conhecimento (Kaufman et al., 2008).

Esta pesquisa demonstra a importância do estudo sobre inteligência e criatividade, na medida em que esses conceitos possuem grande impacto sobre o potencial humano. Pesquisas futuras com amostras de maior tamanho e de outras regiões brasileiras necessitam ser realizadas para ratificar os resultados encontrados. Por outro lado, é necessário também agregar mais informações a fim de observar se a queda do nível de criatividade é percebida pelo próprio indivíduo, por seus pares, professores e familiares. Da mesma forma, estudos com amostras longitudinais são importantes para investigar o percurso do desenvolvimento intelectual e criativo da infância até a velhice.

\section{Agradecimentos}

Os autores agradecem o apoio do Conselho Nacional de Desenvolvimento Técnico e Científico (CNPQ) e da Fundação de Amparo à Pesquisa do Estado de São Paulo (FAPESP) na realização desta pesquisa.

\section{Referências}

Alencar, E. M. L. S. (2007). Criatividade no contexto educacional: três décadas de pesquisa. Psicologia: Teoria e Pesquisa, 23, 45-49.

Alencar, E. M. L. S., \& Fleith, D. S. (2008). Barreiras à promoção da criatividade no ensino fundamental. Psicologia: Teoria e Pesquisa, 24, 59-65.

Aranha, M. A. C. (1997). Creativity in students and its relation to peer perception. Revista Interamericana de Psicologia, 31(2), 309-313. 
Beauchamp, K. D. F., Merle, B., Karnes, M. B., \& Johnson, L. B. (1993). Creativity and intelligence in preschoolers. Gifted Child Quarterly, 37, 113-117.

Camarata, S., \& Woodcock, R. (2006). Sex differences in processing speed: developmental effects in males and females. Intelligence, 34, 231-252.

Cattell, R. B. (1971). Abilities, their structure, growth and action. Boston: Houghton Miffin.

Chiodi, M., \& Wechsler, S. M. (2009). Comparação das baterias de inteligência WJ-III e WISC-III. Avaliação Psicológica, 8(3), 15-25.

Davis, G., \& Belcher, T. L. (1999). How shall creativity be measured? Torrance tests, RAT, Alpha Biographical and IQ. In G. Puccio \& M. C. Murdock (Orgs.), Creativity assessment: readings and resources (pp. 231-239). Buffalo: Creative Education Foundation.

Fleith, D. S., \& Alencar, E. M. L. S. (2005). Escala sobre clima de criatividade em sala de aula. Psicologia: Teoria e Pesquisa, 21, 85-91.

Fleith, D. S., \& Alencar, E. M. L. S. (2006). Percepção de alunos do ensino fundamental quanto ao clima de sala de aula para criatividade. Psicologia em Estudo, 11, 513-521.

Furnham, A., Batey, M., Anand, K., \& Manfield, J. (2008). Personality, hypomania, intelligence and creativity. Personality and Individual Differences, 44, 1060-1069.

Gardner, H. (1999). Intelligence reframed. New York: Basic Books.

Getzels, J. W., \& Jackson, P. M. (1963). The highly intelligent and the highly creative: a summary of research findings. In W. Taylor \& F. Barron (Orgs.), Scientific creativity: its recognition and development (pp. 123-135). New York: Wiley and Sons.

Giglio, Z., Wechsler, S. M., \& Bragotto, D. (Orgs.). (2009). Da criatividade à inovação. Campinas: Papirus.

Guilford, J. P., \& Christensen, P.R. (1973). The one-way relation between creative potential and IQ. Journal of Creative Behavior, 7, 247-252.

Kaufman, J. C., \& Baer, J. (2006). An introduction to the special issue: a tribute to E. Paul Torrance. Creativity Research Journal, 18(1), 1-2.

Kaufman, J. C., Plucker, J. A., \& Baer, J. (2008). Essentials of creativity assessment. New York: John Wiley \& Sons.

Keith, T. Z., Reynolds, M. R., Patel, P. G., \& Ridley, K. P. (2008). Sex differences in latent cognitive abilities ages 6 to 59: evidences from the WoodcockJohnson III tests of cognitive abilities. Intelligence, 36, 502-525.

Kim, K. H. (2006). Is creativity one-dimensional or multidimensional? Analyses of the Torrance Tests of Creative Thinking. Creativity Research Journal, $18,251-259$.

Martinez, A. M. (2002). A criatividade na escola: três direções de trabalho. Linhas Críticas, 8(15), 189-206.

McGrew, K. S. (2005). The Cattell-Horn-Carroll theory of cognitive abilities: past, present and future. In D. P. Flanagan \& P. L. Harrison (Orgs.), Contemporary intellectual assessment (pp. 136-181). New York: The Guilford Press.

McGrew, K. S. (2009). CHC theory and the human cognitive abilities project. Standing on the shoulders of the giants of psychometric intelligence research. Intelligence, 37, 1-10.

McGrew, K., \& Flanagan, D. P. (1998). The intelligence test desk reference (ITDR): Gf-Gc cross battery assessment. Boston: Allyn \& Bacon.

McGrew, K. S., \& Woodcock, R. (2001). Technical manual. Woodcock-Johnson III. Itasca: Riverside Publishing Company.

McKinnon, D. W. (1978). In search of human effectiveness: identifying and developing creativity. Buffalo: Creative Education Foundation.

Mol, D. A. R., \& Wechsler, S. M. (2008). Avaliação de crianças com indicação de dificuldades de aprendizagem pela bateria Woodcock-Johnson III. Psicologia Escolar e Educacional, 12(2), 391-399.

Morais, F., \& Bahia, S. (Orgs.). (2008). Criatividade: conceito, necessidades e intervenção. Braga: Psiquilibrios Edições.

Munford, M. D. (2001). Something old, something new: revisiting Guilford's conception of creative problem solving. Creativity Research Journal, 13, 266-267.

Nakano, T. C., \& Wechsler, S. M. (2006). O percurso da criatividade figural no
Ensino Médio e Superior. Boletim de Psicologia, 125, 205-219.

Nakano, T. C., Wechsler, S. M. (2007). Criatividade: características da produção científica brasileira. Avaliação psicológica, 6(2), 261-270.

Preckel, F., Holling, H., \& Wiese, M. (2006). Relationship of intelligence and creativity in gifted and no gifted students: an investigation of threshold theory. Personality and individual differences, 40, 159-270.

Puccio, G. J., \& Murdock, M. C. (1999). Creativity assessment: readings and resources. Buffalo: Creative Education Foundation.

Raina, M. K. (2006). In my end is my beginning: reflections of the work of a lifetime. Creativity Research Journal, 18, 103-120.

Runco, M. A. (2007). Creativity: theories and themes, research, development and practice. London: Elsevier Academic Press.

Runco, M. A., \& Albert, R. (1986). The threshold theory regarding creativity and intelligence: an empirical study with gifted and no gifted children. The Creative Child and Adult Quarterly, 11, 212-218.

Schrank, F. A. (2005). Woodcock-Johnson III Tests of Cognitive Abilities. In D. P. Flanagan \& P. L. Harrison (Orgs.), Contemporary intellectual assessment: theories, tests and issues (pp. 371-401). New York: The Guilford Press.

Silvia, P. (2008). Another look at creativity and intelligence: exploring higherorder models and probable confounds. Personality and Individual differences, 3, 1012-1021.

Siqueira, L. G. G., \& Wechsler, S. M. (2009). Motivação para aprender e estilos criativos. Educação Temática Digital, 10(3), 70-82.

Sternberg, R. (1999). Handbook of creativity. New York: Cambridge University Press.

Sternberg, R., \& Kaufman, J. (Orgs.). (2001). The evolution of intelligence. Mahawah: Lawrence Erlbaum Associates.

Torrance, E. P. (1965). Rewarding creative behavior. New York: Prentice-Hall.

Torrance, E. P. (1966). Torrance tests of creative thinking. Lexington: Personne Press.

Torrance, E. P. (1990). The Torrance Tests of Creative Thinking. norms-technical manual Figural (streamlined) - Forms A and B. Bensenville: Scholastic Testing Service.

Torrance, E. P., \& Ball, O. (1984). Streamlined scoring and norms for figural form A_and B. Bensenville: Scholastic Testing Service.

Torrance, E. P., Ball, O., \& Safter, T. (1990). Torrance tests of creative thinking: streamlined scoring guide Figural $A$ and B. Benseville: Scholastic Testing.

Torrance, E. P., \& Safter, H. T. (1999). Making the creative leap beyond. Buffalo: Creative Education Foundation Service.

Torre, S. D. L., \& Violant, V. (Orgs.). (2006). Compreender y evaluar la creatividad (Vol. 1). Pavia: Aljibe.

Vincent, A., Decker, B., \& Munford, M. (2002). Divergent thinking, intelligence and expertise: a test of alternative models. Creativity Research Journal, 14, 163-178.

Wallach, M. A., \& Kogan, N. (1965). Modes of thinking in young children: a study of the creativity-intelligence distinction. New York: Holt, Rinehart \& Winston.

Wechsler, S. M. (2004a). Avaliação da criatividade por figuras: Teste de Torrance. Campinas: LAMP/IDB.

Wechsler, S. M. (2004b). Avaliação da criatividade por palavras: Teste de Torrance. Campinas: LAMP/IDB.

Wechsler, S. M. (2006). Validity of the Torrance tests of creative thinking to the Brazilian culture. Creativity Research Journal, 18, 15-25.

Wechsler, S. M. (2009). Avaliação da criatividade: possibilidades e desafios. In C. Hutz (Org.), Avanços e polêmicas em avaliação psicológica (pp. 93-127). São Paulo: Casa do Psicólogo.

Wechsler, S., \& Richmond, B. (1984). Influências da dotação intelectual e criativa no ajustamento em sala de aula. Arquivos Brasileiros de Psicologia, 36(2), 138-147.

Wechsler, S. M., \& Schelini, P. W. (2006). Bateria de habilidades cognitivas Woodcock-Johnson III: validade de construto. Psicologia: Teoria e Pesquisa, 22, 287-295. 
Wechsler, S. M., Vendramini, C. M., \& Schelini, P. W. (2007). Adaptação brasileira dos testes verbais da Bateria Woodcock-Johnson III. Revista Interamericana de Psicologia, 41, 285-294.

Wechsler, S. M., Nunes, C. S., Schelini, P. W., Pasian, S. R., Homsi, S. V., Moretti,
L., Anache, A. A. (no prelo). Brazilian adaptation of the Woodcock-Johnson III tests of cognitive abilities. School Psychology International.

Woodcock, R. W., McGrew, K. S., \& Mather, N. (2001). Woodcock-Johnson III. Itasca: Riverside.

Solange Muglia Wechsler, Ph.D em Psicologia pela University of Georgia (Athens, Georgia, USA), é professora titular de Psicologia na Pontifícia Universidade Católica de Campinas (PUC-Campinas) e diretora do Laboratório de Avaliação e Medidas Psicológicas. Endereço para correspondência: Curso de Pós-graduação em Psicologia, PUC-Campinas, Campus II, Avenida John Boyde Dunlop, s/n, Jardim Ipaussurama, CampinasSP. CEP: 13.059-900. Tel/Fax: (19) 3343-6891/3258-6429. E-mail: wechsler@lexxa.com.br

Maiana Farias Oliveira Nunes, doutora em Psicologia com ênfase em Avaliação Psicológica pela Universidade São Francisco (USF), é pós-doutoranda na Universidade Federal do Rio Grande do Sul (UFRGS) e professora do curso de Psicologia da Faculdade Avantis-SC. E-mail:maiananunes@mac.com

Patrícia Waltz Schelini, doutora em Psicologia como Profissão pela Pontifícia Universidade Católica de Campinas (PUC-Campinas), é professora adjunta no Departamento de Psicologia da Universidade Federal de São Carlos (UFSCar).E-mail: pws@ufscar.br

Adriana Aparecida Ferreira de Souza, mestre em Psicologia Escolar pela Pontifícia Universidade Católica de Campinas (PUC-Campinas), é professor na Universidade de Mogi das Cruzes (UMC). E-mail: adr_ferreira@ hotmail.com

Dejenane Aparecida Pascoal Pereira, doutora em Psicologia Profissão e Ciência pela Pontifícia Universidade Católica de Campinas (PUC-Campinas), é psicóloga da Prefeitura Municipal de São Sebastião-SP. E-mail: pereiradap@uol.com.br 